\title{
The Experience Sampling Method-Evaluation of treatment effect of escitalopram in IBS with comorbid panic disorder
}

\author{
Lisa Vork $^{1}$ (D) | Zlatan Mujagic ${ }^{1}$ (D) | Marjan Drukker ${ }^{2}$ | Daniel Keszthelyi $^{1}$ | \\ José M. Conchillo ${ }^{1}$ | Martine A. M. Hesselink ${ }^{1}$ | Jim van Os ${ }^{2,3,4}$ | Ad A. M. Masclee ${ }^{1}$ | \\ Carsten Leue $^{2}$ | Joanna W. Kruimel ${ }^{1}$
}

\begin{abstract}
${ }^{1}$ Division of Gastroenterology-Hepatology, Department of Internal Medicine, NUTRIM School of Nutrition and Translational Research in Metabolism, Maastricht University Medical Center, Maastricht, The Netherlands

${ }^{2}$ Department of Psychiatry and Psychology, Maastricht University Medical Center, Maastricht, The Netherlands

${ }^{3}$ Department of Psychiatry, Brain Centre Rudolf Magnus, Utrecht University Medical Center, Utrecht, The Netherlands

${ }^{4}$ King's College London, King's Health Partners, Department of Psychosis Studies, Institute of Psychiatry, London, UK
\end{abstract}

\section{Correspondence}

Lisa Vork, Division of GastroenterologyHepatology, Department of Internal Medicine, Maastricht University Medical Center, Maastricht, The Netherlands. Email: I.vork@maastrichtuniversity.nl

\begin{abstract}
Background: Confirming treatment response in clinical trials for irritable bowel syndrome (IBS) is challenging, due to the lack of biomarkers and limitations of the currently available symptom assessment tools. The Experience Sampling Method (ESM) might overcome these limitations by collecting digital assessments randomly and repeatedly during daily life. This study evaluated differences in change in abdominal pain between real-time (ie, ESM) and retrospective (ie, Gastrointestinal Symptom Rating Scale [GSRS] and an end-of-day symptom diary) measurements, using data of an RCT on escitalopram vs placebo in patients with IBS and comorbid panic disorder.

Methods: Twenty-nine IBS patients with comorbid panic disorder were included in a 6-month RCT. The GSRS, diary, and ESM were completed at baseline $(t=0)$ and after $3(t=3)$ and 6 months $(t=6)$. Linear mixed models were used.

Key results: Experience Sampling Method analyses revealed a significant interaction between escitalopram and time, and ESM abdominal pain scores were 1.4 points lower in the escitalopram group compared to placebo at $\mathrm{t}=6$ (on a 1-to-7 scale; $P=0.021)$. When including the interaction with momentary anxiety, the reduction in abdominal pain scores in escitalopram vs placebo was even more pronounced for higher levels of anxiety. Average GSRS- and end-of-day abdominal pain scores were not significantly different between escitalopram and placebo at $\mathrm{t}=3$ and 6 .

Conclusions \& Inferences: Real-time ESM has the potential to capture treatment response more sensitively compared to a retrospective end-of-day GI symptom diary and the GSRS, by taking into account day-to-day symptom variability as well as momentary factors that might moderate treatment effect, such as anxiety.
\end{abstract}

\section{KEYWORDS}

abdominal pain, Experience Sampling Method, irritable bowel syndrome, momentary symptom assessment, treatment evaluation

Prior presentation of results: These data were previously presented in abstract form and oral presentation at the Digestive Disease Days (DDD) in Veldhoven, the Netherlands (2017). The data were also presented in abstract and poster form at the United European Gastroenterology Week (UEGWeek) in Barcelona, Spain (2017), and at the 3rd meeting of the Federation of Neurogastroenterology and Motility in Amsterdam, the Netherlands (2018).

This is an open access article under the terms of the Creative Commons Attribution-NonCommercial-NoDerivs License, which permits use and distribution in any medium, provided the original work is properly cited, the use is non-commercial and no modifications or adaptations are made.

(C) 2018 The Authors. Neurogastroenterology \& Motility Published by John Wiley \& Sons, Ltd. 


\section{1 | INTRODUCTION}

The main challenge in the management of Irritable Bowel Syndrome (IBS) is the selection of a suitable therapy for the individual patient. Overall, the efficacy of most available treatments is modest, the patient population is heterogeneous, and the pathophysiology of IBS is multifactorial and incompletely understood, limiting the development of new treatments. Furthermore, due to the lack of biomarkers and limitations of the currently available symptom assessment tools, confirming treatment response for new and existing therapies in clinical trials remains challenging.

Sensitive tools for symptom assessment are crucial in the evaluation of treatment effect in IBS populations. ${ }^{1}$ Drug regulatory authorities, both in USA and Europe, recommend the use of endof-day symptom scores for evaluation of treatment efficacy in IBS. However, it is well-known that such retrospective (ie, end-of-day), self-reported measurements have important limitations, such as recall and ecological biases. ${ }^{2,3}$ We have previously demonstrated possible advantages of real-time symptom assessment, using the Experience Sampling Method (ESM) compared to end-of-day reporting. ${ }^{4}$ The ESM is an electronic assessment method, which collects data repeatedly at random moments over the day, during several consecutive days. The assessments focus on the in-themoment physical and mental state of the subject, in the natural environment. ${ }^{3,5}$ Considerable differences between real-time and end-of-day abdominal pain scores were demonstrated in a crosssectional study, raising the question of whether real-time symptom assessment may be superior to retrospective methods in the detection of treatment response in IBS patients. ${ }^{6}$ It is expected that ESM shows an increased sensitivity to detect within-subject change over time, as a result of, among other things, repeated data collection. ${ }^{7-9}$

Irritable bowel syndrome is defined as a disorder of gut-brain interaction ${ }^{10}$ with alterations in serotonin metabolism as a presumed key pathophysiological factor. ${ }^{11-13}$ Antidepressants have been utilized in the treatment of IBS for many years. With regard to the efficacy of antidepressants in IBS, global symptom improvement and increased quality of life as well as a reduction in abdominal pain have been demonstrated for tricyclic antidepressants (TCAs), but not for selective serotonin reuptake inhibitors (SSRIS). ${ }^{14,15}$ Furthermore, overlap between IBS and psychiatric disorders is common in daily practice ${ }^{16-18}$ and IBS patients with psychological comorbidities may constitute the IBS subgroup most prone to respond to neuromodulating therapy.

In our tertiary referral center, the prevalence of comorbid panic disorder in IBS patients is high (up to $50 \%$ of this population). ${ }^{19}$ According to current guidelines for management of anxiety disorders, the firstchoice treatment for panic disorder is SSRIs. ${ }^{20,21}$ However, it is not known whether IBS patients with panic disorder benefit from SSRIs with regard to their IBS symptoms and whether anxiety might moderate treatment response. Therefore, the current randomized controlled trial was developed to examine the effect of the SSRI escitalopram on abdominal pain in IBS patients, diagnosed with comorbid panic disorder. However, due to slow patient inclusion rates, the study has been

\section{Key Points}

- Differences between real-time and retrospective symptom reports have been demonstrated cross-sectionally in irritable bowel syndrome (IBS). This study evaluated differences in treatment evaluation between these methods.

- The real-time Experience Sampling Method (ESM) demonstrated a decrease in abdominal pain after 6 months of escitalopram treatment, related to anxiety, whereas retrospective methods could not find a difference between escitalopram and placebo.

- ESM potentially captures treatment response more sensitively than retrospective reports, by taking into account day-to-day symptom variability and treatment-modulating momentary factors.

prematurely ended. Since the originally intended sample size was not achieved, a firm conclusion on the efficacy of SSRIs in IBS with panic disorder cannot be drawn. Nevertheless, as both real-time and retrospective patient-reported symptom scores were collected before, during, and after the treatment period, the data are suitable to compare treatment response measurements between ESM and retrospective symptom reports.

The primary objective of the current study was to evaluate differences in change in abdominal pain by use of real-time (ie, ESM) and retrospective (ie, Gastrointestinal Symptom Rating Scale [GSRS] and an end-of-day symptom diary) symptom assessment methods, in the escitalopram treatment group vs the placebo group after, respectively, 3 and 6 months of treatment, in patients with IBS and comorbid panic disorder. Secondary, we aimed to evaluate the modulating effects of psychological comorbidity, that is, anxiety, on the effect of escitalopram on abdominal pain, by using ESM.

\section{2 | MATERIALS AND METHODS}

\section{1 | Study design}

The current study uses data of a single-center, double-blind, parallel-group, randomized controlled trial of escitalopram vs placebo for patients with IBS and comorbid panic disorder. The study protocol was approved in 2011 by the medical-ethics committee of Maastricht University Medical Centre (MUMC), Maastricht, the Netherlands, and was registered in the US National Library of Medicine (https://www.clinicaltrials.gov, NCT01551225). The study was executed according to the principles of the revised Declaration of Helsinki (59th WMA General Assembly, Seoul, Republic of Korea, October 2008). All subjects gave their written informed consent prior to participation. 


\section{2 | Study participants and medication}

Study participants were recruited between February 2012 and June 2016, via the MUMC's outpatient clinics of Gastroenterology and the Med-Psych-Centre. The latter is a secondary/tertiary outpatient clinic for the combined consultation of a gastroenterologist and a hospital-psychiatrist, delivering integrated care. Subjects aged 18-70 years, clinically diagnosed with IBS and comorbid panic disorder, were considered eligible. IBS was diagnosed by a gastroenterologist (JK) using the Rome III criteria ${ }^{22,23}$ and, if indicated, GI endoscopy, abdominal imaging, and/or blood, breath or fecal analyses were performed to exclude organic $\mathrm{Gl}$ diseases. The presence of a comorbid panic disorder was diagnosed by a psychiatrist $(\mathrm{CL})$ using the DSM-IV-TR criteria and the Mini-International-Neuropsychiatric-Interview. ${ }^{24}$ Details on exclusion criteria and subject monitoring during the trial are described in Supporting Information 1.

Included subjects were randomly assigned to one of the two treatment groups (ie, escitalopram or placebo) in a 1:1 ratio and started with an initial dose of study medication of $5 \mathrm{mg}$ once a day. This was increased to $10 \mathrm{mg}$ once daily after the first week and could be gradually increased to a maximum of $20 \mathrm{mg}$ once daily, depending on the individual response to treatment in terms of self-reported symptom improvement. Further information on randomization procedures and study medication can be found in Supporting Information 1.

\section{3 | Study procedures}

Three standardized measurement moments, that is, baseline $(t=0)$, after three months $(t=3)$, and after 6 months $(t=6)$, were implemented in the six-month study period. At baseline, questionnaires were completed on demographics, lifestyle, and medication use. At baseline and the two follow-up moments, validated retrospective symptom questionnaires, a 14-day end-of-day symptom diary, and a 7-day ESM period were completed.

\subsection{1 | Symptom questionnaires}

Validated questionnaires that were completed at baseline and after 3 and 6 months included the Gastrointestinal Symptom Rating Scale (GSRS) and the Rand 36-item Short-Form Health Survey (SF-36). The GSRS is a 15-item self-reported scale, evaluating GI symptom severity based on a single measurement, with a recall period of one week. All individual items are scored on a 7-point Likert scale ( $1=$ not at all to $7=$ extremely) and are subsequently clustered into five domains (abdominal pain, reflux, indigestion, diarrhea, and constipation); higher scores indicate more severe symptoms. ${ }^{25,26}$ The SF-36 was used to evaluate general quality of life, resulting in a score for physical (PCS) as well as mental (MCS) quality of life (ie, higher scores indicate better quality of life)..$^{27,28}$

\subsection{2 | Symptom diary}

A 14-day end-of-day symptom diary was used to evaluate GI symptom severity on a daily basis over 14 consecutive days. GI symptoms (ie, abdominal pain, abdominal discomfort, abdominal bloating, flatulence, constipation, and diarrhea) were scored using a 5-point Likert scale ( $1=$ not at all to $5=$ extremely) at the end of each day, so considering a recall period of one day.

\subsection{3 | Experience Sampling Method}

On seven consecutive days during the 14-day diary period, the electronic ESM was used for momentary assessment of $\mathrm{Gl}$ symptoms and the in-the-moment affective state. ESM assessments were performed on a palmtop computer; the device sent out an auditory signal ("beep") at 10 random moments between 7.30 AM and 10.30 PM, and subjects completed an electronic questionnaire following each beep. The ESM-questionnaire was repeated in the same order at all measurement moments, and all questions were scored by using a 7point Likert scale $(1=$ not at all to $7=$ extremely). In contrast to the retrospective symptom questionnaires and end-of-day diary, there is no recall but ESM measures real-time experiences.

\subsection{Statistical analyses}

All analyses were performed using Stata, version 13.1 (Stata Corporation, College Station, TX, 2009). Baseline demographical characteristics and symptom severity scores are presented as medians (Q1; Q3) for continuous data and as proportions for categorical data. The primary outcome measures were abdominal pain severity, as measured by using the ESM, the end-of-day symptom diary, and the abdominal pain-domain of the GSRS (GSRS-AP). ESM and endof-day diary data had a three-level structure (level 1: repeated measurements at each ESM assessment [ESM] and at the end of each day [diary], respectively; level 2: time [ $\mathrm{t}=0,3,6$ ]; level 3: subject); GSRS data had a two-level structure (level 1: time $[t=0,3,6]$; level 2: subject). The primary ESM analysis included all ESM data of the subjects that completed three valid ESM periods (ie, at least 23 out of 70 [1/3] assessments per period). This rule of thumb for validity of ESM periods has been common standard in ESM protocols, in order to take into account the fact that including data of subjects that complete too few assessments, may distort overall study findings. ${ }^{29,30}$ An ESM sensitivity analysis included all subjects that collected ESM data at all three measurement moments. All analyses with end-of-day diary or GSRS as the outcome measure included all subjects that collected data at the three measurement moments (ie, excluding two dropouts). A $P$-value of 0.05 was considered statistically significant.

\section{Change in abdominal pain}

To analyze whether the change in abdominal pain over time was different in escitalopram compared to placebo, mixed linear models with unstructured covariance, random slopes for treatment and time, and including the treatment*time interaction were used. Separate models were used for the three different assessment methods, with abdominal pain scores (ie, ESM, end-of-day diary, and GSRS) as the dependent variable. All main effects were included 
as a random slope, however, in some models, the random slope for time and/or treatment had to be removed because the model did not reach conversion. All non-significant interaction terms $(\alpha=0.05)$ were removed from the models top-down. Regression coefficients in each strata were obtained by using the Stata lincom post-estimation command in the final models.

In order to evaluate a possible modulating effect of anxiety, anxiety scores were additionally included in the model with ESM abdominal pain scores as the dependent variable. Both two-way interactions anxiety*escitalopram, anxiety*time, and escitalopram*time, and the three-way interaction anxiety*escitalopram*time as well as the main effect of anxiety were included. Momentary anxiety scores assessed using ESM (on a 1-to-7 point scale) were used. Using the Stata postestimation command lincom, regression coefficients are shown for scores 1 (no anxiety), 2 (low anxiety), 3 (mild anxiety), and 4 (moderate anxiety), as subjects did not frequently score anxiety higher than 4 .

\subsection{1 | Sample size}

We originally intended to include 80 subjects (ie, 40 in each group) in this RCT, aiming to evaluate the effect of escitalopram vs placebo on abdominal pain in IBS patients with comorbid panic disorder. However, patient inclusion rate remained lower than expected and the study was prematurely ended with group sizes of 15 and 14 for

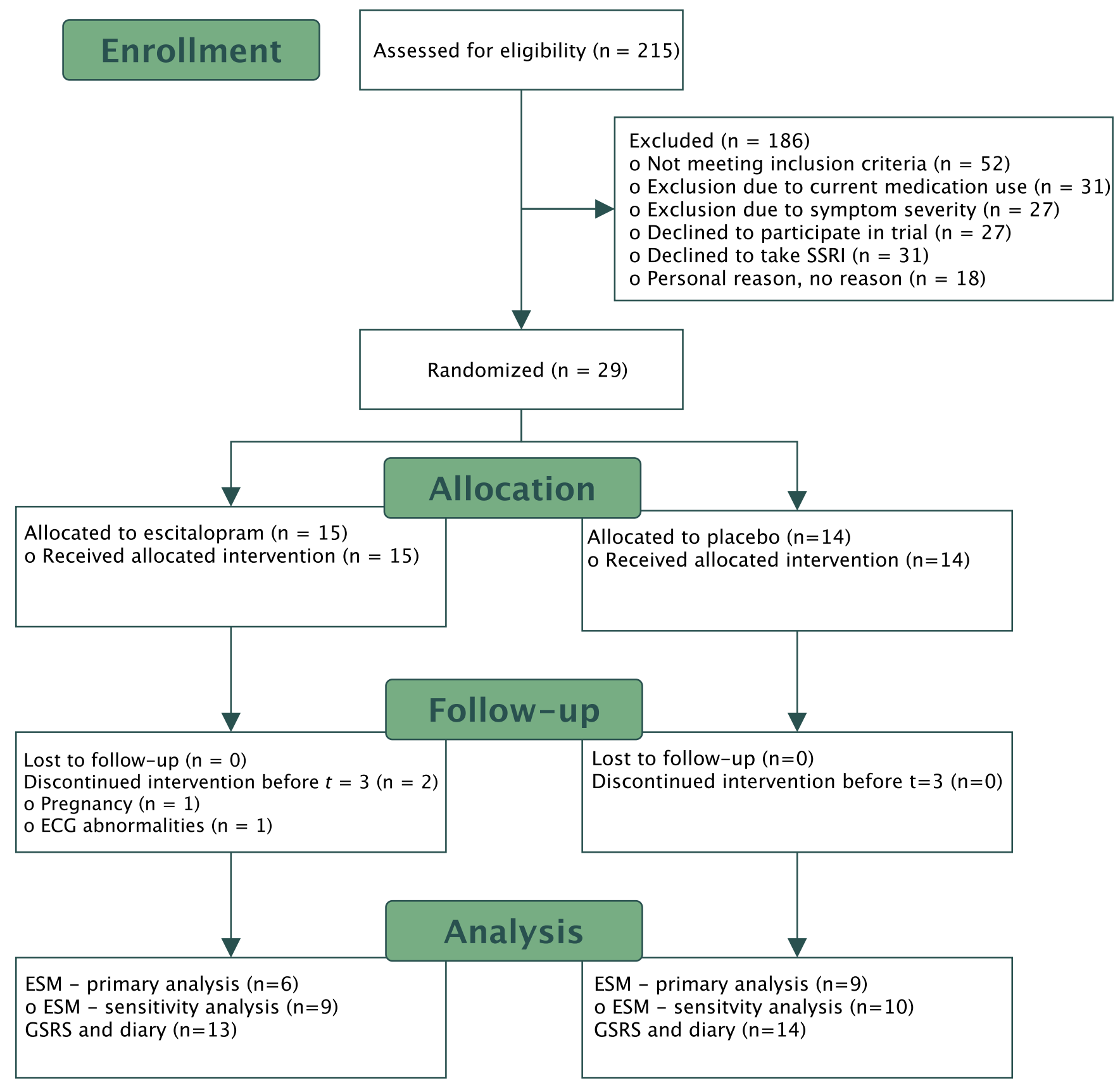

FIGURE 1 Flow chart of subject enrollment 
TAB LE 1 Demographical and clinical patient characteristics for escitalopram and placebo treatment groups, at baseline $(t=0)$

\begin{tabular}{|c|c|c|}
\hline & \multicolumn{2}{|l|}{ Baseline, $\mathrm{t}=0$} \\
\hline & $\begin{array}{l}\text { Escitalopram } \\
(n=15)\end{array}$ & Placebo $(n=14)$ \\
\hline Female sex, n (\%) & $12(80.0)$ & $9(64.3)$ \\
\hline Age, median (Q1; Q3) & $39.00(25.00 ; 55.00)$ & $25.50(23.00 ; 46.00)$ \\
\hline BMI, median (Q1; Q3) & $26.93(25.99 ; 29.40)$ & $25.40(23.53 ; 28.04)$ \\
\hline \multicolumn{3}{|l|}{ IBS subtype, n (\%) } \\
\hline IBS-D & $6(40.0)$ & $6(42.9)$ \\
\hline IBS-C & $5(33.3)$ & $0(0.0)$ \\
\hline IBS-M & $4(26.7)$ & $8(57.1)$ \\
\hline IBS-U & $0(0.0)$ & $0(0.0)$ \\
\hline \multicolumn{3}{|l|}{ GSRS, median (Q1; Q3) } \\
\hline Abdominal pain & $3.33(2.33 ; 4.33)$ & $3.67(2.67 ; 4.00)$ \\
\hline Reflux syndrome & $2.50(1.00 ; 3.00)$ & $1.50(1.00 ; 4.00)$ \\
\hline Diarrhea syndrome & $3.67(3.00 ; 5.67)$ & $2.67(2.33 ; 4.00)$ \\
\hline $\begin{array}{l}\text { Constipation } \\
\text { syndrome }\end{array}$ & $4.00(2.00 ; 4.00)$ & $3.33(2.33 ; 4.33)$ \\
\hline $\begin{array}{l}\text { Indigestion } \\
\text { syndrome }\end{array}$ & $3.75(2.75 ; 4.50)$ & $3.38(2.25 ; 4.25)$ \\
\hline \multicolumn{3}{|c|}{ End-of-day diary, median (Q1; Q3) } \\
\hline Abdominal pain & $2.71(1.93 ; 2.86)$ & $2.39(2.21 ; 3.50)$ \\
\hline Discomfort & $2.71(2.31 ; 3.36)$ & $2.75(2.43 ; 3.57)$ \\
\hline Bloating & $2.57(1.64 ; 3.08)$ & $2.71(1.78 ; 3.29)$ \\
\hline Diarrhea & $1.29(1.00 ; 1.69)$ & $1.15(1.00 ; 1.50)$ \\
\hline Constipation & $1.15(1.00 ; 169)$ & $1.46(1.14 ; 2.23)$ \\
\hline \multicolumn{3}{|l|}{ SF-36, median (Q1; Q3) } \\
\hline $\begin{array}{l}\text { Mental Composite } \\
\text { Score (MCS) }\end{array}$ & $46.84(41.10 ; 53.85)$ & $43.30(30.75 ; 51.41)$ \\
\hline $\begin{array}{l}\text { Physical Composite } \\
\text { Score (PCS) }\end{array}$ & $41.39(32.66 ; 47.84)$ & $40.86(34.27 ; 46.17)$ \\
\hline
\end{tabular}

GSRS scores are presented for five subscales. End-of-day diary scores are presented as average scores over 14 days, based on the sum of individual scores per day. SF-36 scores are presented for two subscales.

escitalopram and placebo, respectively. Therefore, we cannot draw a firm conclusion on the efficacy of escitalopram, but we here exploratory describe differences in the measurement of treatment response between real-time and retrospective assessment methods.

\section{3 | RESULTS}

\section{1 | Study population}

During the enrollment period, 215 IBS patients with comorbid panic disorder were screened for eligibility, of whom twenty-nine (13.5\%) were included in the study (see Figure 1). Twenty-one (72.4\%) were female, and the median age was 37 years (IQR: 24-49). IBS subtype distribution was as follows: 12 diarrhea-predominant (IBS-D), five constipation-predominant (IBS-C), and 12 mixed type (IBS-M).
Fifteen subjects were randomized to the escitalopram group (12 female [80.0\%]; median age 39 years) and 14 to the placebo group (nine female [64.3\%]; median age 25.5 years). Abdominal symptom scores at baseline, according to the GSRS and end-of-day symptom diary, were similar in both groups, just as the SF-36 scores. Baseline characteristics are shown in Table 1. Two subjects (6.9\%; both from the escitalopram group) dropped out before $t=3$ months, for reasons of unplanned pregnancy $(n=1)$ and development of ECG abnormalities on routine ECG before dose elevation of escitalopram $(n=1)$. Since the primary aim of the current study was not to evaluate the effect of escitalopram vs placebo, but to compare outcomes of different methods for treatment response measurement, these two subjects with incomplete data were not included in the analyses.

Of the total study population, fifteen subjects $(51.7 \%$; six escitalopram, nine placebo) completed a valid number of ESM assessments (ie, at least 23 out of 70 assessments per period) at all three time points. The data of these subjects were used for the primary ESM analysis. Baseline characteristics of this subgroup were comparable to those of the total study population. Information on side effects during the trial can be found in Supporting Information 1.

\subsection{Evaluation of treatment response using ESM}

Twenty-one subjects (72.4\%) agreed to complete the ESM questionnaires, of whom 15 (71.4\%) collected a valid number of assessments (ie, at least 23 out of 70 assessments per period) at all three time points. In the primary ESM analysis including ESM data of these 15 subjects, a significant two-way interaction escitalopram*time was found $\left(\chi^{2}: 101.22, d f=2, P<0.001\right)$, revealing that abdominal pain decreased more over time in the escitalopram group than the placebo group. Furthermore, the lincom post-estimation command showed that abdominal pain scores were significantly lower in the escitalopram group compared to placebo at $\mathrm{t}=6(\mathrm{~B}=-1.425, P=0.021$; on a 1-to-7 scale). In the ESM sensitivity analysis, including data from all 19 subjects that completed ESM, the two-way interaction was significant as well $\left(\chi^{2}: 31.08, d f=2, P<0.001\right)$, but abdominal pain scores were not significantly different between escitalopram and placebo at $t=3$ $(\mathrm{B}=-0.280, P=0.631)$ and $\mathrm{t}=6(\mathrm{~B}=-0.783, P=0.181)$ (Table 2$)$. Mean ESM abdominal pain scores for escitalopram and placebo at all three time points are shown in Figure 2. The differences in change in abdominal pain scores between escitalopram and placebo at $\mathrm{t}=3$ and 6 months are shown in Table 2.

\subsection{1 | Interaction with anxiety}

Additionally, anxiety levels were also included in this model. The three-way interaction anxiety*escitalopram*time was revealed not statistically significant $\left(\chi^{2}: 4.45, d f=2, P=0.108\right)$. However, since we a priori expected a limited power to show this interaction, we performed the lincom post-estimation command to obtain the regression coefficients for the different strata despite the $p$-value above 
alpha. At $\mathrm{t}=6$ months, average abdominal pain score was 1.414 point lower $(P=0.040)$ for escitalopram compared to placebo when there was no anxiety $(1)$ and this difference increased to $1.601(P=0.024)$ for low anxiety (2), $1.788(P=0.026)$ for mild anxiety (3), and 1.975 $(P=0.036)$ for moderate anxiety (4). Results are shown in Table 3.

\section{3 | Evaluation of treatment response using GSRS and end-of-day diary}

In models assessing GSRS-AP and the end-of-day abdominal pain score, we did not find a significant two-way interaction between escitalopram and time, showing that the association between escitalopram and abdominal pain was similar at all time points using retrospective assessments (Table 2). Mean abdominal pain scores for escitalopram and placebo at all three time points are shown in Figure 2, for both methods.

\section{4 | DISCUSSION}

In the present exploratory study, we evaluated the possible advantages of real-time symptom assessment using ESM, in quantifying treatment response, when compared to retrospective symptom measurements using an end-of-day diary and the GSRS. Hereto, data from a prematurely ended randomized controlled trial, developed to evaluate the effect of escitalopram vs placebo on abdominal pain in IBS patients with comorbid panic disorder, were used. Hence, no beneficial effect of escitalopram over placebo in reducing abdominal pain in subjects with IBS and comorbid panic disorder was found, when evaluated by conventional, retrospective symptom assessment methods (ie, GSRS and end-of-day abdominal pain scores). However, when momentarily assessed by the real-time electronic ESM, a significant decrease in abdominal pain was observed in escitalopram as compared to placebo, after 6 months of treatment. Due to the exploratory nature of the used method, we cannot draw a firm conclusion on the effect of SSRIs on abdominal pain in IBS patients based on the presented results, however, the current study underlines the difference in outcome between retrospective and repeated in-the-moment measurements. Furthermore, these results show that with the high number of repeated measures and the real-time assessment of symptoms, ESM may have the potential to detect within-subject changes over time with higher sensitivity, yielding higher analytical power in small numbers of study participants, when compared to conventional retrospective questionnaires.

Differences between retrospective and real-time assessments have previously been demonstrated in IBS populations. Reporting of peak abdominal pain rather than average scores has been described, for weekly or daily retrospective measurement compared to momentary abdominal pain assessment. ${ }^{4,31}$ This phenomenon can be attributed to recall bias, suggesting that real-time assessments might be more accurate with regard to abdominal pain evaluation in a cross-sectional study design. The present study is the first to evaluate differences in outcome of treatment effect over time, between retrospective and momentary assessments of abdominal pain. 
(A) Escitalopram

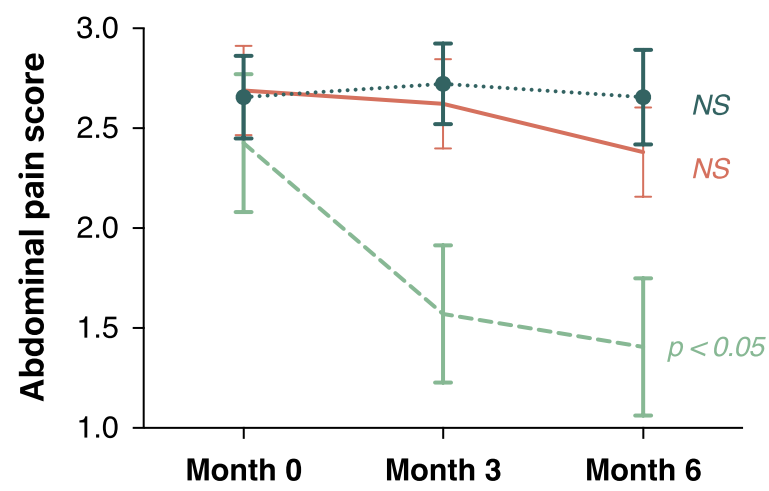

(B) Placebo

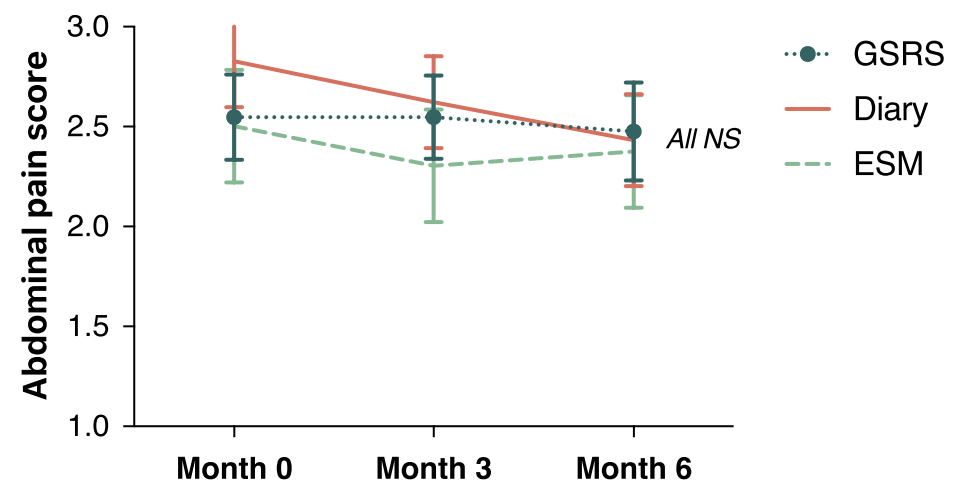

FIGURE 2 Mean abdominal pain scores at $t=0$ months (baseline), $t=3$ months, and $t=6$ months for the escitalopram group ( $\mathrm{A}$ ) and placebo group (B), separately for GSRS, end-of-day diary, and ESM (on a 1-to-5 point scale). Change in abdominal pain scores over time tested using linear mixed models. NS, no significant change in abdominal pain scores after 3 and 6 months. $P<0.05$, significant change in abdominal pain scores after 6 months

Apart from eliminating recall bias, ESM has the advantage of capturing within-subject variability over time, as a result of repeated data collection. In the context of evaluating treatment effect, this is beneficial as it leads to an increased sensitivity to detect within-subject change over time..$^{7-9}$ Regarding the ESM analyses in the current study, it should be noted that a significant decrease in abdominal pain was only found in the primary analysis which included the ESM data of subjects that completed three valid ESM periods, that is, at least 23 out of 70 assessments per period, underlining the importance of collecting sufficient repeated assessments when using ESM. Our findings confirm results from earlier trans-diagnostic ESM data obtained from patients with functional or unexplained somatic symptoms and psychiatric comorbidity treated at an academic hospital-psychiatry outpatient ward with regard to ESM's sensitivity to detect change. ${ }^{32}$

Given the recognized efficacy of escitalopram in panic disorder treatment, ${ }^{33,34}$ we hypothesized an interaction between anxiety, escitalopram, and time in the model evaluating the change in abdominal pain. Even though no significant three-way interaction term could be found, a more pronounced decrease in abdominal pain in the escitalopram group could be demonstrated for subjects with higher levels of momentary anxiety as compared to subjects with lower levels of anxiety. These results demonstrate the potential of ESM to evaluate the effect of momentary factors possibly modulating symptom formation and/or treatment response and add value to the field by integrating somatic and psychological assessment at the same time.

The current study is based on data of a prematurely ended RCT, which was set up to evaluate the effect of an SSRI on abdominal pain in IBS patients with comorbid panic disorder. The intended number of subjects to be enrolled was 80 , while after over 4 years, only 29 subjects were included. This was $13.5 \%$ of all eligible patients approached for participation within this period. Given the availability of the drug (SSRI) in regular clinical care and the anticipation of a possible placebo treatment for 6 months, many patients decided not to participate in the trial. This illustrates the difficulty of performing a high-quality placebo-controlled RCT for the evaluation of drug efficacy in IBS patients, or any other chronic

TAB LE 3 Difference in ESM abdominal pain scores between escitalopram and placebo for ESM-anxiety levels of 1 (no anxiety), 2 (low anxiety), 3 (mild anxiety), and 4 (moderate anxiety) (on a 1-to-7 Likert scale), respectively, tested using linear mixed models. Results shown for $\mathrm{t}=0,3$, and 6 months

\begin{tabular}{|c|c|c|c|c|c|c|c|c|c|}
\hline \multirow{2}{*}{$\begin{array}{l}\text { ESM } \\
\text { abdominal } \\
\text { pain }\end{array}$} & \multicolumn{3}{|l|}{ Month 0 (baseline) } & \multicolumn{3}{|l|}{ Month 3} & \multicolumn{3}{|l|}{ Month 6} \\
\hline & Estimate $(95 \% \mathrm{CI})$ & SE & $P$-value & Estimate $(95 \% \mathrm{Cl})$ & SE & $P$-value & Estimate $(95 \% \mathrm{Cl})$ & SE & $P$-value \\
\hline $\begin{array}{l}\text { No anxiety } \\
\text { (1) }\end{array}$ & $-0.117(-1.467 ; 1.233)$ & 0.689 & 0.865 & $-1.179(-2.528 ; 0.170)$ & 0.688 & 0.087 & $-1.414(-2.762 ;-0.066)$ & 0.688 & 0.040 \\
\hline $\begin{array}{l}\text { Low } \\
\text { anxiety (2) }\end{array}$ & $0.085(-1.268 ; 1.437)$ & 0.690 & 0.902 & $-0.909(-2.265 ; 0.447)$ & 0.692 & 0.189 & $-1.601(-2.994 ;-0.208)$ & 0.711 & 0.024 \\
\hline $\begin{array}{l}\text { Mild } \\
\text { anxiety (3) }\end{array}$ & $0.286(-1.130 ; 1.702)$ & 0.722 & 0.692 & $-0.640(-2.070 ; 0.791)$ & 0.730 & 0.381 & $-1.788(-3.359 ;-0.216)$ & 0.802 & 0.026 \\
\hline $\begin{array}{l}\text { Moderate } \\
\text { anxiety (4) }\end{array}$ & $0.488(-1.044 ; 2.019)$ & 0.781 & 0.533 & $-0.370(-1.934 ; 1.194)$ & 0.798 & 0.643 & $-1.975(-3.820 ;-0.129)$ & 0.942 & 0.036 \\
\hline
\end{tabular}

Estimate indicates the difference between ESM abdominal pain scores in the escitalopram group vs the placebo group. A negative estimate indicates a lower abdominal pain score in the escitalopram group compared to placebo. SE, standard error.

The model included a three-way interaction term for anxiety ${ }^{*}$ escitalopram*time: $\chi^{2}: 4.45(2), P=0.108$. 
condition, especially in cases of comorbidities. Furthermore, well conducted RCTs are costly and time-consuming and may put participants at risk, due to the unknown nature of treatment effects. Therefore, methods that may reduce the number of required study participants in clinical trials are highly needed. ESM provides the opportunity to identify significant treatment effects in a lower number of study participants, compared to the currently available retrospective questionnaires, by gathering a high number of repeated measures and more reliable data that are not affected by recall and ecological biases.

A strength of the current study is that symptoms were concurrently reported using ESM, an end-of-day diary, and the GSRS during an RCT, offering the opportunity to directly compare outcome of treatment effect between real-time and retrospective measurements. Furthermore, the inclusion of a well-characterized tertiary IBS population with pronounced comorbid anxiety, in which both IBS and anxiety symptoms were repeatedly measured using ESM, enabled us to evaluate the potential of ESM to take into account momentary factors modulating symptom formation and treatment effect.

A limitation of this study is that not all included subjects agreed to complete ESM, which decreased the sample size in the ESM analyses and might have induced selection bias. As discussed previously, the use of ESM might overcome this limitation concerning sample size by taking into account multiple repeated measurements in a single patient and thereby increasing the power to detect change over time. With regard to possible selection bias, we found no differences in demographical characteristics and gastrointestinal and psychological symptom severity between subjects that completed ESM and subjects that did not. A drawback of ESM can be the fact that the method is time-consuming, which might lead to a lack of compliance. On the other hand, due to the repeating character, it is expected that ESM results are not easily influenced by missing data. Furthermore, we took into account the risk of low compliance by excluding subjects that completed too few ESM assessments from the primary analyses. With regard to the study participants, IBS subtype distribution is different between the treatment groups in that no subjects with the constipation predominant subtype were present in the placebo group. However, as this study focuses on abdominal pain as the main outcome measure and subtype distribution is the same for all measurement methods, our primary results are most likely not affected by this difference in subtype distribution. Nevertheless, given the exploratory nature of this study and the fact that ESM has not yet been validated as an evaluation tool in the context of clinical trials evaluating abdominal pain, our results need replication in order to draw a firmer conclusion on the advantages of ESM over retrospective methods in quantifying treatment response in IBS patients.

\section{5 | CONCLUSION}

In conclusion, the current study demonstrates a potential benefit of repeated in-the-moment measurements as compared to retrospective assessments, in the evaluation of treatment effect in IBS patients. Given the exploratory nature of the study, no firm conclusion can be drawn with regard to the effect of SSRIs on abdominal pain in IBS patients with comorbid panic disorder. Nevertheless, by taking into account day-to-day symptom variability as well as momentary factors that might moderate treatment effect, such as psychological symptoms, ESM has shown the potential to capture treatment response more sensitively compared to a retrospective end-of-day GI symptom diary and the GSRS.

\section{ACKNOWLEDGMENTS}

Gratitude is hereby expressed to D. Marcus, T. Schagen, J. Scheepers, C. Brandts, M. Kameh Khosh, T. Klaassen, R. Kliest, and B. Swampillai for their contributions to the study. Furthermore, we would like to thank Y. Keulemans and C. Hoge for being available as independent physicians during this study.

\section{DISCLOSURES}

No competing interests to declare.

\section{AUTHOR CONTRIBUTIONS}

Study concept and design: JK, CL, AM, JO. Data collection and processing: LV, ZM, JK, CL, JC, MH. Statistical analysis: LV, MD, ZM. Manuscript writing: LV. Constructive review of manuscript: ZM, MD, JK, CL, DK, AM, JO. All authors approved the final manuscript.

\section{ORCID}

Lisa Vork (iD http://orcid.org/0000-0001-7316-3191

Zlatan Mujagic iD http://orcid.org/0000-0001-5211-0381

\section{REFERENCES}

1. Mujagic Z, Keszthelyi D, Aziz Q, et al. Systematic review: instruments to assess abdominal pain in irritable bowel syndrome. Aliment Pharmacol Ther. 2015;42(9):1064-1081.

2. Shiffman S, Stone AA, Hufford MR. Ecological momentary assessment. Ann Rev Clin Psychol. 2008;4:1-32.

3. Myin-Germeys I, Oorschot M, Collip D, Lataster J, Delespaul P, van Os J. Experience sampling research in psychopathology: opening the black box of daily life. Psychol Med. 2009;39(9):1533-1547.

4. Mujagic Z, Leue C, Vork L, et al. The Experience Sampling Method - a new digital tool for momentary symptom assessment in IBS: an exploratory study. Neurogastroenterol Motil. 2015;27(9):1295-1302.

5. Vork L, Keszthelyi D, Mujagic Z, et al. Development, content validity, and cross-cultural adaptation of a patient-reported outcome measure for real-time symptom assessment in irritable bowel syndrome. Neurogastroenterol Motil. 2018;30(3):e13244

6. van Os J, Verhagen S, Marsman A, et al. The experience sampling method as an mHealth tool to support self-monitoring, self-insight, and personalized health care in clinical practice. Depression and Anxiety. 2017;34(6):481-493. 
7. Verhagen SJ, Hasmi L, Drukker M, van Os J, Delespaul PA. Use of the experience sampling method in the context of clinical trials. Evidence-Based Mental Health. 2016;19(3):86-89.

8. Bolger N, Laurenceau JP. Intensive Longitudinal Methods: An Introduction to Diary and Experience Sampling Research. New York, NY: Guilford Press; 2013.

9. Bolger N, Davis A, Rafaeli E. Diary methods: capturing life as it is lived. Annu Rev Psychol. 2003;54:579-616.

10. Drossman DA, Tack J, Ford AC, Szigethy E, Tornblom H, Van Oudenhove L. Neuromodulators for functional gastrointestinal disorders (disorders of gut-brain interaction): a Rome Foundation Working Team report. Gastroenterology. 2018;154(4):1140-1171.e1141.

11. Lesurtel M, Soll C, Graf R, Clavien PA. Role of serotonin in the hepato-gastrolntestinal tract: an old molecule for new perspectives. Cell Mol Life Sci. 2008;65(6):940-952.

12. Cremon C, Carini G, Wang B, et al. Intestinal serotonin release, sensory neuron activation, and abdominal pain in irritable bowel syndrome. The American Journal of Gastroenterology. 2011;106(7):1290-1298.

13. Thijssen AY, Mujagic Z, Jonkers DM, et al. Alterations in serotonin metabolism in the irritable bowel syndrome. Aliment Pharmacol Ther. 2016;43(2):272-282.

14. Ford AC, Quigley EM, Lacy BE, et al. Effect of antidepressants and psychological therapies, including hypnotherapy, in irritable bowel syndrome: systematic review and meta-analysis. Am J Gastroenterol. 2014;109(9):1350-1365; quiz 1366.

15. Ruepert L, Quartero AO, de Wit NJ, van der Heijden GJ, Rubin G, Muris J. Bulking agents, antispasmodics and antidepressants for the treatment of irritable bowel syndrome. Cochrane Database Syst Rev. 2011(8):CD003460.

16. Drossman DA, Creed FH, Olden KW, Svedlund J, Toner BB, Whitehead WE. Psychosocial aspects of the functional gastrointestinal disorders. Gut. 1999;45(suppl 2):li25-30.

17. Palsson OS, Drossman DA. Psychiatric and psychological dysfunction in irritable bowel syndrome and the role of psychological treatments. Gastroenterol Clin North Am. 2005;34(2):281-303.

18. Ballou S, Keefer L. The impact of irritable bowel syndrome on daily functioning: Characterizing and understanding daily consequences of IBS. Neurogastroenterol Motil. 2017;29(4):e12982.

19. Kruimel J, Leue C, Winkens B, et al. Integrated medical-psychiatric outpatient care in functional gastrointestinal disorders improves outcome: a pilot study. Eur J Gastro Hepatol. 2015;27(6):721-727.

20. Balkom van ALJM, Vliet van IM, Emmelkamp PMG, et al. Multidisciplinaire Richtlijn Angststoornissen. 2013; Versie 1.0 (3e revisie): https://assets-sites.trimbos.nl/docs/cc5b0f4e-9398-452d8792-1453a38bf0a8.pdf, 2013. Accessed 6 June 2017.

21. American Psychiatric Association. Practice Guideline for the Treatment of Patients with Panic Disorder. Second edition ed: American Psychiatric Association; 2010.

22. Longstreth GF, Thompson WG, Chey WD, Houghton LA, Mearin F, Spiller RC. Functional bowel disorders. Gastroenterology. 2006;130(5):1480-1491.

23. Drossman DA. Rome III: the new criteria. Chin J Dig Dis. 2006;7(4): 181-185.
24. Diagnostic and Statistical Manual of Mental Disorders (DSM)-IV-TR. 4th ed. Washington, DC: American Psychiatric Association; 2000.

25. Svedlund J, Sjodin I, Dotevall G. GSRS - a clinical rating scale for gastrointestinal symptoms in patients with irritable bowel syndrome and peptic ulcer disease. Dig Dis Sci. 1988;33(2): 129-134.

26. Revicki DA, Wood M, Wiklund I, Crawley J. Reliability and validity of the Gastrointestinal Symptom Rating Scale in patients with gastroesophageal reflux disease. Qual Life Res. 1998;7(1):75-83.

27. Farivar SS, Cunningham WE, Hays RD. Correlated physical and mental health summary scores for the SF-36 and SF-12 Health Survey, V.I. Health Qual Life Outcomes. 2007;5:54.

28. McHorney CA, Ware Jr JE, Raczek AE. The MOS 36-Item ShortForm Health Survey (SF-36): II. Psychometric and clinical tests of validity in measuring physical and mental health constructs. Med Care. 1993;31(3):247-263.

29. Palmier-Claus JE, Myin-Germeys I, Barkus E, et al. Experience sampling research in individuals with mental illness: reflections and guidance. Acta Psychiatr Scand. 2011;123(1):12-20.

30. Delespaul P. Assessing schizophrenia in daily life the experience sampling method. UPM, Universitaire Pers Maastricht, Maastricht University; 1995.

31. Weinland SR, Morris CB, Hu Y, Leserman J, Bangdiwala SI, Drossman DA. Characterization of episodes of irritable bowel syndrome using ecological momentary assessment. Am J Gastroenterol. 2011;106(10):1813-1820.

32. Verhagen S, Berben JA, Leue C, et al. Demonstrating the reliability of transdiagnostic mHealth Routine Outcome Monitoring in mental health services using experience sampling technology. PLoS ONE. 2017;12(10):e0186294.

33. Bakker A, van Balkom AJ, Spinhoven P. SSRIs vs. TCAs in the treatment of panic disorder: a meta-analysis. Acta Psychiatr Scand. 2002;106(3):163-167.

34. Stahl SM, Gergel I, Li D. Escitalopram in the treatment of panic disorder: a randomized, double-blind, placebo-controlled trial. J Clin Psychiatry. 2003;64(11):1322-1327.

\section{SUPPORTING INFORMATION}

Additional supporting information may be found online in the Supporting Information section at the end of the article.

How to cite this article: Vork L, Mujagic Z, Drukker M, et al. The Experience Sampling Method-Evaluation of treatment effect of escitalopram in IBS with comorbid panic disorder. Neurogastroenterol Motil. 2019;31:e13515. https://doi. org/10.1111/nmo.13515 